\title{
X-ray investigation of buried SiGe islands for devices with strain-enhanced mobility
}

N. Hrauda, J. J. Zhang, J. Stangl, A. Rehman-Khan, G. Bauer, M. Stoffel, O. G. Schmidt, V. Jovanovich, and L. K. Nanver

Citation: Journal of Vacuum Science \& Technology B: Microelectronics and Nanometer Structures Processing, Measurement, and Phenomena 27, 912 (2009); doi: 10.1116/1.3056178

View online: https://doi.org/10.1116/1.3056178

View Table of Contents: http://avs.scitation.org/toc/jvn/27/2

Published by the American Institute of Physics 


\title{
X-ray investigation of buried SiGe islands for devices with strain-enhanced mobility
}

\author{
N. Hrauda, a) J. J. Zhang, J. Stangl, A. Rehman-Khan, and G. Bauer \\ Johannes Kepler University, Altenberger Strasse 69, 4040 Linz, Austria \\ M. Stoffel and O. G. Schmidt \\ IWF Dresden, Helmholtzstrasse 20, 01069 Dresden, Germany
}

\author{
V. Jovanovich and L. K. Nanver \\ Technical University Delft, Stevinweg 1, Delft 2628, The Netherlands
}

(Received 18 August 2008; accepted 1 December 2008; published 30 March 2009)

\begin{abstract}
In this work self-organized SiGe islands are used as stressors for Si capping layers, which later act as carrier channels in field effect transistors. To be able to address individual islands and to obtain a sufficiently narrow distribution of their properties, the SiGe islands are grown by molecular beam epitaxy on prepatterned $\mathrm{Si}$ substrates, with a regular two-dimensional array of pits. This combination of lithographic patterning and self-assembled island growth combines the advantages of both approaches and leads to very homogeneous island shape, size, and chemical composition. For processing, 4 in. wafers are used, and fields with pit periods between 600 and $1000 \mathrm{~nm}$ are defined by optical lithography. After growth of a Si buffer layer several monolayers of Ge are deposited, leading to island formation (dome or barn shaped) in the pits. Subsequent Si capping is performed at a low substrate temperature of $300{ }^{\circ} \mathrm{C}$ to avoid intermixing and shape changes of the buried islands. The Ge distribution in the buried islands and the strain distribution in the islands and the surrounding $\mathrm{Si}$ matrix are assessed by $\mathrm{x}$-ray diffraction experiments, combined with three-dimensional model simulations using finite elements. Tensile strain values in the Si cap up to $8 \times 10^{-3}$ can be achieved using this approach, which is difficult to achieve using other methods without introduction of dislocations. (C) 2009 American Vacuum Society. [DOI: 10.1116/1.3056178]
\end{abstract}

\section{INTRODUCTION}

In semiconductor industry strained $\mathrm{Si}$ is one of the options to enhance the performance of metal-oxide semiconductor (MOS) devices. ${ }^{1}$ Schmidt and Eberl ${ }^{2}$ proposed to use selfassembled $\mathrm{SiGe}$ islands as stressors for $\mathrm{Si}$ capping layers, which allow to reach higher strain values as compared to approaches based on pseudomorphic Si layers on relaxed SiGe alloy buffers. ${ }^{3}$ The tensile strain in the Si capping layers lifts the degeneracy of the six $\Delta$ valleys in the Si conduction band, splits those into $\Delta_{2}$ and $\Delta_{4}$ valleys, and induces a downward shift in energy of the $\Delta_{2}$ valleys. For sufficiently large tensile strains, only the $\Delta_{2}$ valleys are populated and the electrons in these two valleys determine with their in plane, i.e., their transverse mass, the mobility, which is consequently higher than in the unstrained state. In addition, we follow an approach where the $\mathrm{SiGe}$ islands themselves are removed at later stages of processing, leaving Si bridges to reduce leakage currents. A schematic sketch of such a sample is shown in Fig. 1(a) and the final device in Fig. 1(b), according to the concept of the disposable dot field effect transistor (d-DOTFET). ${ }^{4}$ For this sort of devices several important processing steps are necessary. In particular, twodimensional ordered $\mathrm{SiGe}$ islands arrays have to be grown on (001) Si substrates. Such ordered arrays have been realized in the past either by growing through appropriate masks on $\mathrm{Si}$ or by using buried stressors, or by deposition of Ge on pit

${ }^{\text {a) }}$ Electronic mail: nina.hrauda@jku.at patterned Si substrates. ${ }^{5-14}$ For our investigations we achieve dot ordering by growth on pit patterned substrates. The (001) silicon substrate is patterned by optical lithography and reactive ion etching to produce a two-dimensional (2D) array of pits, in which later SiGe islands will form. This step enables not only further processing steps for which each island has to be addressed but leads also to a narrow distribution of island properties such as shape, size, and composition. ${ }^{9,11}$

Due to the larger lattice constant of Ge (about $4.2 \%$ larger than that of Si for pure $\mathrm{Ge}$ ), dome or barn-shaped islands form in the pits during growth on silicon substrate, depending on the number of monolayers deposited. ${ }^{9}$ Domes as well as barns are multifaceted islands; domes with an aspect ratio

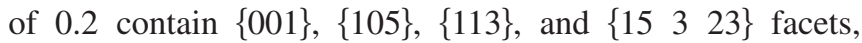
whereas the steeper barns (aspect ratio of 0.32) additionally show $\{111\},\{20423\}$, and $\{23420\}$ facets. These islands are then overgrown by a silicon layer at low temperatures to avoid intermixing and shape changes of the islands, which would lead to a loss of strain in the silicon cap. Subsequent capping with strained $\operatorname{SiN}_{x}$ increases the strain within the silicon cap immediately and helps keeping the tensile strain even when the buried island is removed later. Patterned stressors such as carbon films on top of GaAs quantum wells have been employed previously by Kash et al. ${ }^{15,16}$ to realize one-dimensional quantum wire structures with stresses in the carbon layer up to $500 \mathrm{MPa}$ which is a comparable value to the $445 \mathrm{MPa}$ stress achieved for $\mathrm{SiN}_{x}$ layers in this work. 


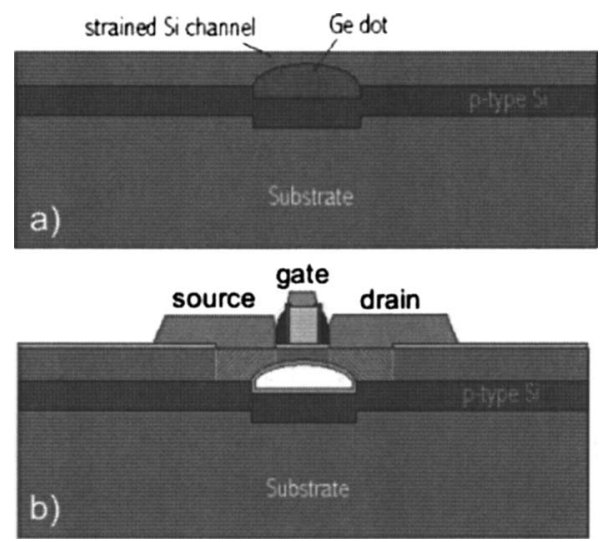

FIG. 1. Sketch of the planned device structure with the SiGe dot still present (a) and the final device using strained silicon as carrier channel with the SiGe dot removed (b).

In the d-DOTFET concept after the deposition of $\mathrm{SiN}_{x}$, deep trenches aligned to the spacing of the pattern are etched to access the islands for the final etching step. ${ }^{4}$ Here the islands are removed using a selective etchant leaving freestanding silicon bridges behind. Based on finite element simulations, Vastola et al. ${ }^{17}$ calculated the strain distribution in the Si bridges before and after removal of the buried islands.

In this article experimental investigations using x-ray diffraction techniques ${ }^{18}$ on the strain status of the samples representing the processing steps up to the $\mathrm{SiN}_{x}$ capping are summarized.

\section{EXPERIMENTAL}

\section{A. Sample growth}

As substrate, 4 in. Si (001) wafers were used. Regular 2D arrangements of pits were defined by optical lithography and reactive ion etching. First, either 18 or $36 \mathrm{~nm}$ of silicon buffer was grown with the substrate temperature ramped from 360 to $500{ }^{\circ} \mathrm{C}$. After deposition of the Si buffer the pits remain with the shape of an inverted and truncated pyramid with $\left\{\begin{array}{lll}1 & 1 & 10\end{array}\right\}$ side facets. Then several monolayers of Ge were deposited at a temperature of $700{ }^{\circ} \mathrm{C}$. Whereas the growth of 5-6 ML Ge results in dome-shaped islands, more than $8 \mathrm{ML} \mathrm{Ge}$ lead to formation of barn-shaped ones. As next step, the dots are capped with a $50 \mathrm{~nm}$ thick silicon layer, which serves as channel material for the final device. This capping is done at low temperatures $\left(300{ }^{\circ} \mathrm{C}\right)$ to maintain the shape of the dots and avoid intermixing, which would lead to strain relaxation. ${ }^{18}$ From atomic force microscopy (AFM) measurements it was observed that the Si cap itself takes over more or less the shape of the buried dot so that facets are still visible. Subsequent $\mathrm{SiN}_{x}$ capping is performed by plasma-enhanced chemical vapor deposition. With this method, amorphous films are deposited at rather low temperatures $\left(<400{ }^{\circ} \mathrm{C}\right)$.

\section{B. X-ray measurements}

Whereas AFM measurements give information about the shape and size of the islands, high resolution x-ray diffraction is used to determine the average chemical composition as well as the strain state of the island. ${ }^{18}$ An alternative method for the determination of only the Ge content variation in SiGe films and $\mathrm{SiGe}$ islands is based on selective chemical etching techniques reported by $\mathrm{Wu}$ and Hull, ${ }^{19}$ Katsaros et al., ${ }^{20}$ and Leite et al. ${ }^{21}$ In general, reciprocal space maps were recorded in coplanar (high angle-) geometry around the symmetric (i.e., the 004) and several asymmetric Bragg peaks (113 and 224).

For the first sample series containing mainly uncapped islands (domes and barns) and one sample with capped domes, x-ray measurements have been performed at the beamline BW2, Hasylab, Hamburg. For data collection a 2D charge coupled device camera was used, so by performing 1:2 relative scans around a Bragg peak (where relative to the aligned peak position, the range for the detector angle $\theta$ is twice as large as the incident angle $\omega$ ) three-dimensional (3D) data were collected in a single scan. For each sample, reciprocal space maps around the symmetric (004) and the asymmetric (113) reflection were recorded at a wavelength of $1.7712 \AA$.

In these maps the intensities are plotted as a function of the coordinates $Q_{z}$ (along the [001] direction) and $Q_{x}$ (along [110]) where

$$
Q_{x}=2 \pi / \lambda[\cos (2 \theta-\omega)-\cos \omega]
$$

and

$$
Q_{z}=2 \pi / \lambda[\sin \omega+\sin (2 \theta-\omega)] .
$$

The second series of samples (domes capped with $50 \mathrm{~nm} \mathrm{Si}$, one capped additionally with $55 \mathrm{~nm} \mathrm{SiN}_{x}$ ) was measured at the beamline ID10B, ESRF, Grenoble. Reciprocal space maps of the scattered intensities around the (224) Bragg peak for these two samples are shown in Fig. 2. Panel (a) shows data on a sample with domes of $240 \mathrm{~nm}$ diameter and a period of $900 \mathrm{~nm}$, capped with $50 \mathrm{~nm}$ Si. Below, a measurement from a similar sample capped additionally with $55 \mathrm{~nm}$ $\mathrm{SiN}_{x}$ is shown. The sharp signal in the middle of the plots represents the intensities stemming from the silicon bulk material and is used as internal standard. The signal on the left side below results from the $\mathrm{SiGe}$ dots. Due to the small amount of material and the inhomogenous composition, it is a smeared intensity distribution rather than a sharp peak. The shape of this signal strongly depends on the shape, size, and the distribution of Ge within those islands. Comparing these two maps, a slight shift of the SiGe signal to the left, i.e., to lower $Q_{x}$ values, can be seen in the lower part of Fig. 2. This indicates a larger in-plane lattice constant of the SiGe compound, therefore a higher degree of relaxation within the island itself, which again leads to a higher tensile strain in the pseudomorphic silicon cap above.

The determination of strain and composition is performed in two steps: from the peak positions in reciprocal space, a rough guess on average strain and composition is obtained 


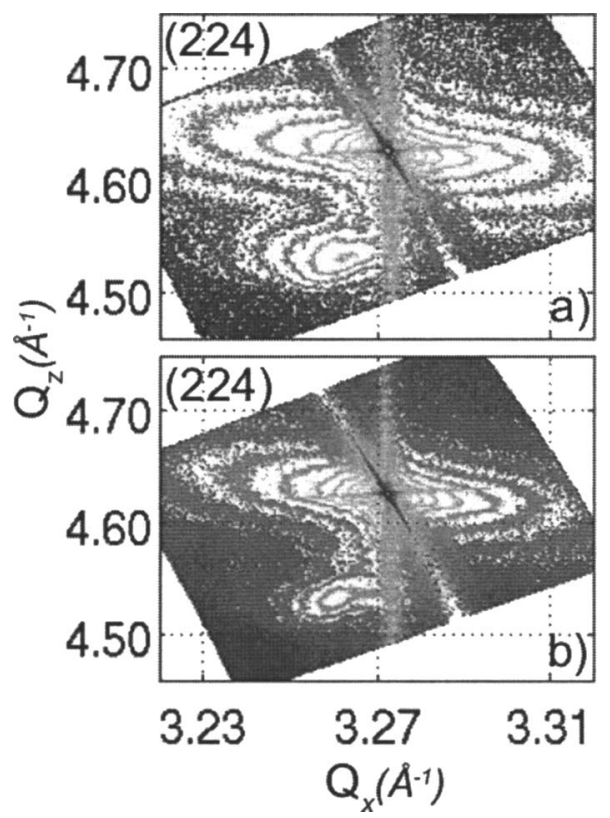

FIG. 2. Reciprocal space maps around the (224) Bragg peak for two samples grown under the same conditions, without (upper panel, sample s3023) and with (lower panel, sample s3025) an additional cap of $55 \mathrm{~nm}$ thick $\mathrm{SiN}_{x}$.

using Bragg's law. For uncapped islands the scattering signal due to the $\mathrm{SiGe}$ island is smeared over a larger region of reciprocal space for domes as well as for barns. This is due to the larger strain relaxation which occurs in uncapped islands compared to capped ones. Therefore the determination of the "peak position" leads to a large error range. As an example reciprocal space maps of the (113) Bragg peak of a sample containing uncapped domes can be seen in Fig. 3,

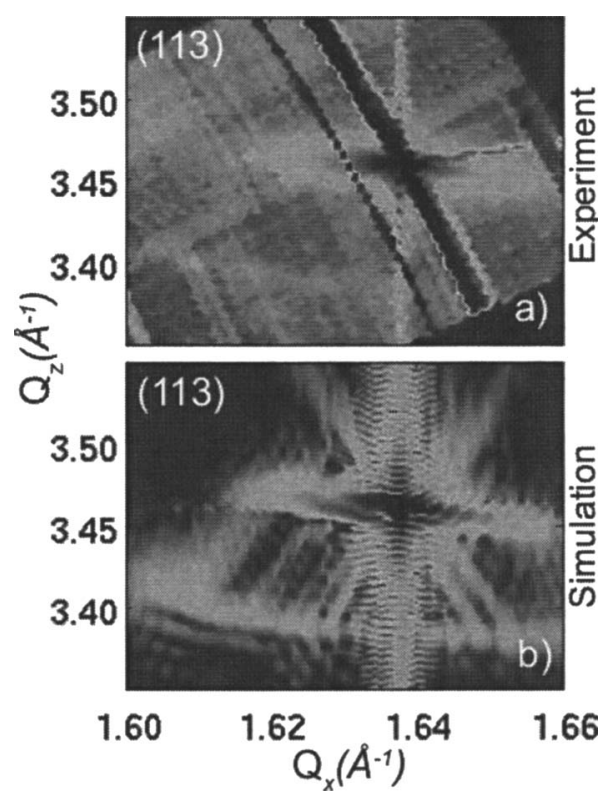

FIG. 3. Experimental data [panel (a)] and simulation [panel (b)] on a sample with uncapped domes. The faint signal below the silicon (113) Bragg peak originates from the $\mathrm{SiGe}$ islands. where panel (a) shows the experiment and panel (b) the respective simulation.

Signals measured from capped islands are sharper, the maximum can be determined more accurately. However, in this case the hydrostatic pressure that is applied by the surrounding Si matrix to the buried islands leads to an underestimation of the Ge content.

Therefore, the data obtained by this crude approach are used only as an input for a more accurate determination of strain and composition, based on finite element method (FEM) calculations ${ }^{22}$ and simulation of scattered $\mathrm{x}$-ray intensity distribution. It turned out that the average Ge contents calculated from the peak positions are about 5\% too low. Detailed information about the results as well as the according fits will be given in the next section.

\section{SIMULATIONS}

The periodic arrangement of SiGe islands leads to a periodic satellite pattern in the scattered intensity in reciprocal space. However, due to the large period of the pattern $(600-1000 \mathrm{~nm})$, the satellite distance in reciprocal space is very small and was not resolved in our experiments. Rather, the envelope function of the scattered intensities is measured, which corresponds well to the scattering of an individual island, multiplied by the number of illuminated islands (about $10^{5}$ ). Hence for our 3D simulations a $\mathrm{Si}$ substrate block with a single island in a $\left[\begin{array}{lll}1 & 1 & 10\end{array}\right]$ pit was considered. Special care was given to the exact shape of the structure, which not only contains all the nominal facets of dome- and barn-shaped islands but also the surrounding, for example, the pit where the island is located, the shape of the capping layer, and the diameter and depth of the trench. All these properties were carefully derived from AFM measurements. Several examples for model shapes are shown in Fig. 4.

The base of the SiGe structure, which contains not only the island itself but also the part of the pit filled with $\mathrm{SiGe}$, is situated at the origin of the coordinate system. This provides an efficient way to apply analytic functions to describe the germanium gradients in all three spatial directions $(x, y$, and $z)$. To calculate the strain distribution in epitaxial structures, usually the whole geometry is defined, then the respective lattice mismatches are assigned to the individual model parts, and then the strain state is calculated of the equilibrium of mutual straining of the finite elements of the model.

For more advanced processing steps, e.g., the additional capping with silicon nitride, the etching of trenches, and the removal of the island itself, attention has to be paid on the way the model is set up as there are different growth processes involved.

The growth of the silicon buffer, the SiGe islands, and also the capping with silicon is done epitaxially, in this case by molecular beam epitaxy. Thus a model for a SiGe island capped with $\mathrm{Si}$ can be set up in one step, as described above. This is not the case anymore, when an additional $\mathrm{SiN}_{x}$ capping layer is applied. For this material the growth mode and therefore the crystalline properties of the material itself are different. Whereas the $\mathrm{Si}$ and $\mathrm{SiGe}$ structures are perfectly 


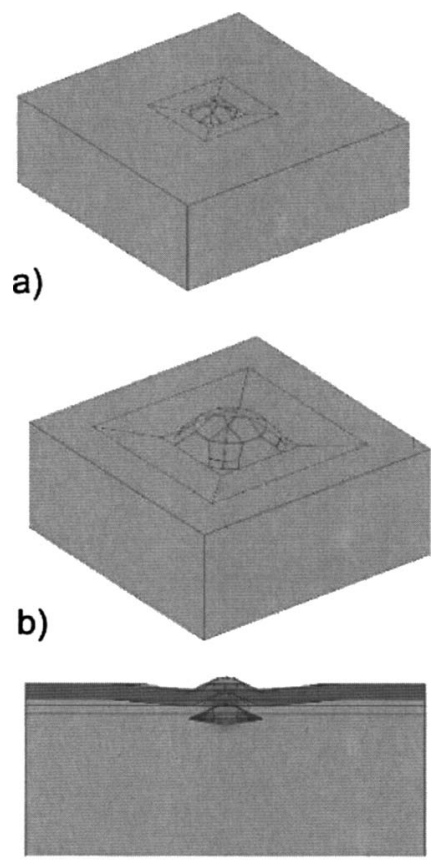

c)

FIG. 4. Examples of the models used for FEM calculation. The uppermost image shows a substrate block with an uncapped dome-shaped island in a pit. In the middle part an island with barn shape is shown. The lowest part of the figure illustrated the setup of a model with the Si bulk, the SiGe structure, Si capping layer, and a layer of silicon nitride on top.

crystalline without defects, the silicon nitride is amorphous, and it is deposited on top of the strained $\mathrm{Si} / \mathrm{SiGe}$ structure. As there is no epitaxial relationship, the $\mathrm{SiN}_{x}$ layer has a strain state independent of the underlying sample. One may assume that it is strain-free at the growth temperature and has some strain after cooling down due to different thermal expansion of the layer and the material below. Hence in FEM modeling first the strain of the material below is calculated as usual. Taking this as a starting point, the $\operatorname{SiN}_{x}$ layer is then added, with a certain initial strain taking into account the thermal strain, and the strain distribution resulting from the mutual straining of $\mathrm{SiN}_{x}$ and the material below is calculated in the second step.

A model of the final structure- $\mathrm{a}$ freestanding silicon bridge with the island removed-contains four steps: the first step contains, as mentioned before, the silicon matrix (bulk, cap) and the $\mathrm{SiGe}$ island. In the second step the $\mathrm{SiN}_{x}$ layer is added. The two following levels contain first the etching of the trenches to access the buried islands which are then removed in the final step. For each of those processing steps the strain distribution of the proceeding step is used as a starting point, the geometry is changed accordingly (etching away material is modeled by inactivating parts of the geometry), and the resulting FEM structure equilibrated again.

Depending on the growth conditions, silicon nitride can be grown in a way that it applies either tensile or compressive stress to the layers below. To optimize the $\mathrm{SiN}_{x}$ stressor layer, a test series of blank $\mathrm{Si}$ wafers was capped with $\mathrm{SiN}_{x}$ deposited by plasma-enhanced chemical vapor deposition us- ing different $\mathrm{NH}_{3}$ flows, $\mathrm{rf}$ powers, and deposition temperatures in the range from 300 to $350{ }^{\circ} \mathrm{C}$. Stress values were determined from the exact position of the (004) Bragg peak at several positions of the wafer, using the shift of angles to calculate the curvature of the wafer and thus the stress applied by the $\mathrm{SiN}_{x}$ layer. Under optimum conditions a maximum stress of $445 \mathrm{MPa}$ was achieved and those were used to cap the wafers with the buried $2 \mathrm{D}$ arrays of islands. This value was then used for FEM calculations.

To finally deduce the Ge composition profile and strain distribution, data of the strain distribution in the FEM model are used to calculate x-ray diffraction (XRD) scattering patterns and compared to the measured patterns. The Ge profiles are then varied until simulation and experiment show a good match. With this fitting approach, the Ge distribution within the $\mathrm{SiGe}$ islands is determined as well as the strain distribution in the islands and the surrounding $\mathrm{Si}$.

Generally, for the uncapped samples of the first growth series measured at BW2, germanium contents ranging from about $14 \%$ to $47 \%$ (domes) and $36 \%$ to $46 \%$ (barns) were obtained from the peak positions of the scattered x-ray intensities. In the 3D FEM simulation the germanium content is increasing along growth direction $z$ following a square function with a certain starting value at the bottom and a maximum Ge content at the apex. To account for the lateral composition changes, along the $x$ - and $y$-directions, a $\mathrm{Ge}$ decrease toward the rim of the island was applied. For the uncapped sample S2922 of the first series a vertical Ge gradient from $33 \%$ to $53 \%$ and lateral gradients a with Ge decreasing according to a square root function was used. The results are shown in Fig. 3(b).

The second growth series was aimed at the study of the influence of $\mathrm{SiN}_{x}$ stressors, thus these samples were grown with Si caps without growth interruption. It turned out that for this series the Ge content of the dome-shaped islands was slightly higher than of those in the first series, with a germanium content increase along $z$ following a square function with a starting value of $45 \%$ at the bottom and a maximum $\mathrm{Ge}$ content at the apex of $65 \%$. The range for the gradients in lateral direction is $30 \%$. We emphasize that the strain distribution in the $\mathrm{Si}$ cap changes considerably due to "restraining" of the partially relaxed islands as compared to the uncapped case. The Ge distribution and the strain distributions are shown in Fig. 5. The average Ge content for this model is $40 \%$, which is about $5 \%$ higher than the value derived from the peak position alone, without simulations.

For barn-shaped islands, no sample containing capped islands has been processed so far. For such a sample (S2926) a germanium distribution following a square root function with $30 \%$ at the bottom of the island and a maximum value of $50 \%$ at the apex yielded a reasonable good fit of measured and calculated x-ray data.

For samples with nitride cap, the two-step fitting procedure explained above has to be applied. Figure 6 shows the comparison of measured and simulated reciprocal space maps around the (224) Bragg peak for a sample (s3025) containing domes capped with $50 \mathrm{~nm}$ silicon and additionally 


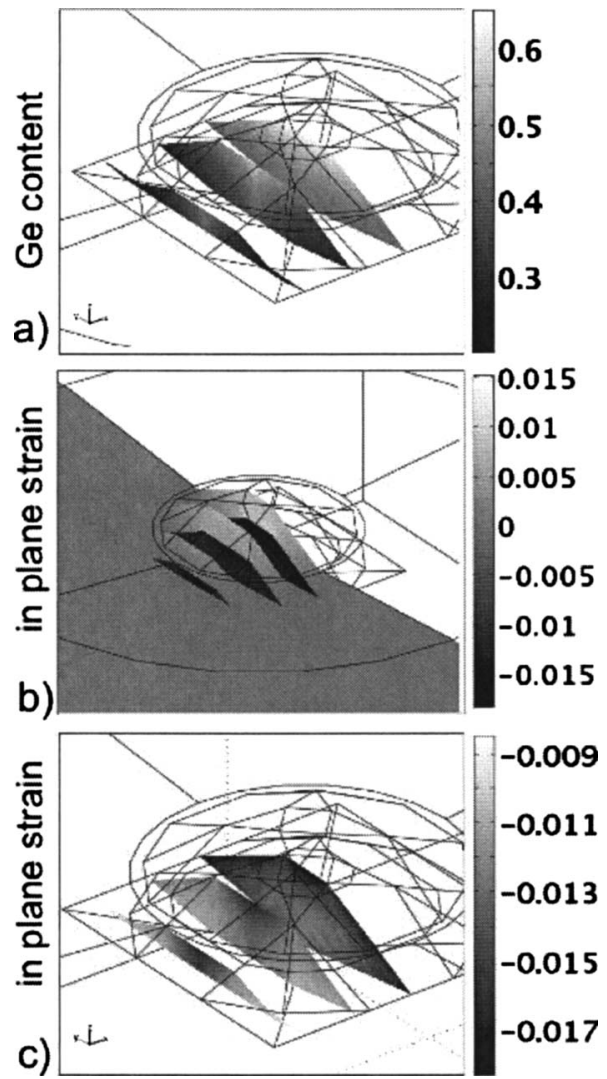

FIG. 5. (a) FEM simulation for sample s3023 (SiGe domes with $240 \mathrm{~nm}$ diameter, capped with $50 \mathrm{~nm}$ silicon). The topmost plot shows the 3D Ge distribution with $\mathrm{Ge}$ content increasing along $z$ and decreasing along the $x$ and $y$ directions. (b) Strain distribution both in the SiGe island (negative values indicate compressive strain with respect to the relaxed compound) and the silicon capping layer (tensile strained). (c) same as (b) with smaller range of plotted strain values to make details of strain distribution within the island visible.

$55 \mathrm{~nm} \mathrm{SiN}{ }_{x}$. As initial stage, the results for sample s3023 (same structure without $\mathrm{SiN}_{x}$ cap) were used as the first step. In the second step the $\mathrm{SiN}_{x}$ cap is added as described before. For this layer, a compressive initial stress value of about $445 \mathrm{MPa}$ was derived from curvature measurements of wafers grown under the same conditions. Figure 7(a) shows a 2D map of the strain distribution calculating using FEM (cut through the center of the island). The line plot in panel (b) shows the in-plane strain along a vertical line from the bottom of the SiGe structure to the top. Strain values are given with respect to the relaxed state of the particular materialsthe silicon bulk and capping layer, which is in case of the bulk mainly relaxed, the SiGe structure, which is compressively strained and the nitride layer, to which in this case a compressive initial strain was assigned. In the tensile silicon part, it can be seen that the strain decreases slightly to a value of about $0.7 \%$ compared to the value of $0.8 \%$ obtained for the sample without nitride cap.

\section{DISCUSSION AND SUMMARY}

The strain change in the Si cap layer due to the nitride is counterintuitive: for a compressive initial stress in the ni-

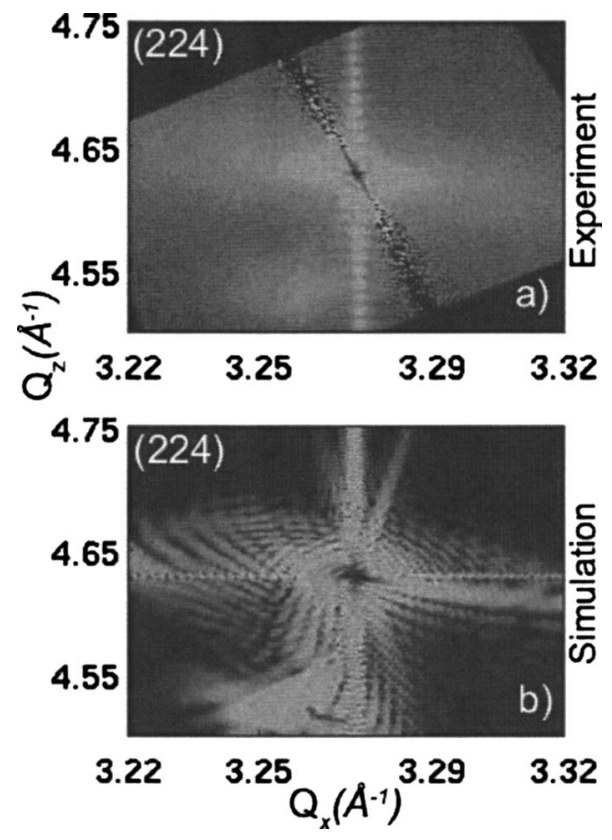

FIG. 6. Comparison of measurement [panel (a)] and simulation [panel (b)] for sample s3025, SiGe domes capped with $50 \mathrm{~nm} \mathrm{Si}$ and $55 \mathrm{~nm} \mathrm{SiN}$. As input concerning the Ge distribution, the results obtained for a sample grown under the same conditions but without $\operatorname{SiN}_{x}$ layer were used.

tride, one would assume that the nitride expands upon equilibration, hence drags the Si cap along and increases the tensile strain in the $\mathrm{Si}$ cap. To investigate the situation systematically, we have performed a series of simulations with different initial stress values of the nitride. Figure 8

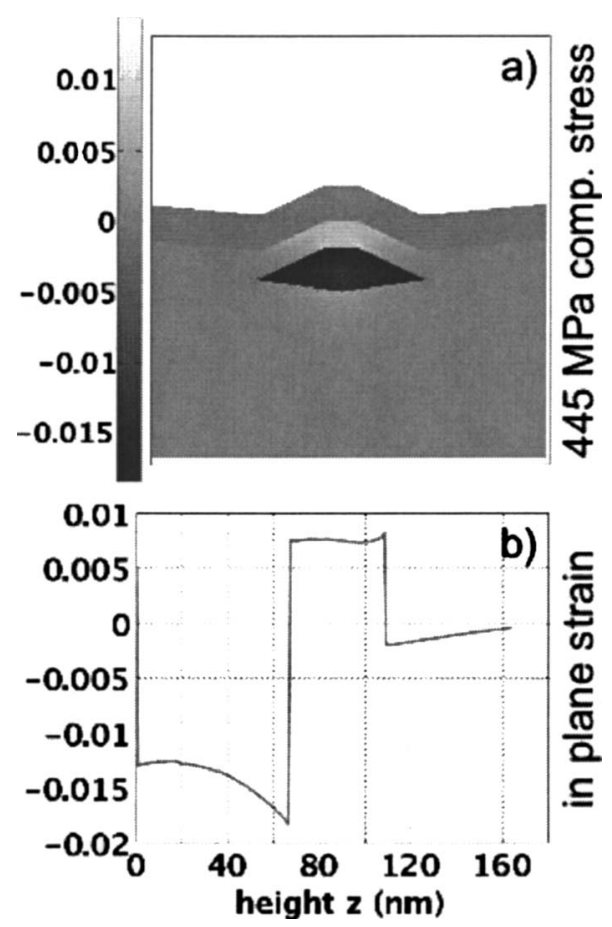

FIG. 7. (a) 2D strain map and (b) line plot through the center of the island, displaying the in-plane strain of the $\mathrm{SiGe}$ island and the $\mathrm{Si}$ and $\mathrm{SiN}_{x}$ capping layers. Here, the full 3D Ge distribution is considered (see text). 


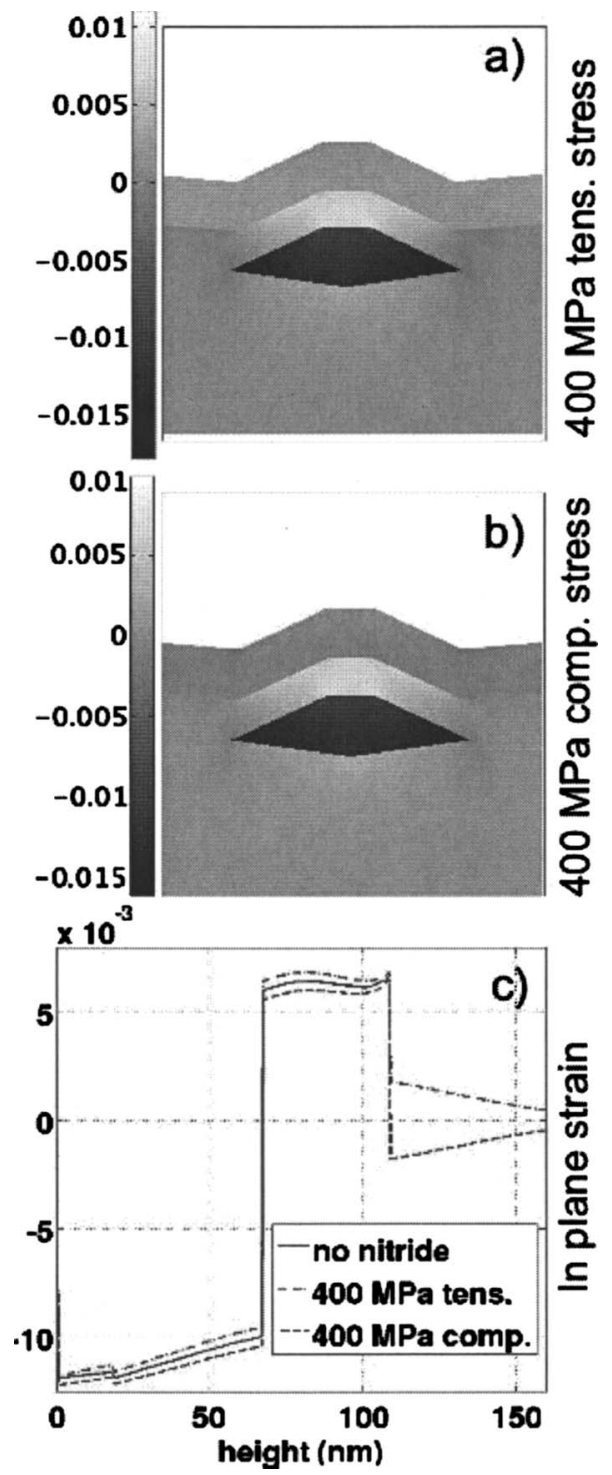

FIG. 8. Comparison of FEM simulations with tensile (a) and compressively (b) strained $\mathrm{SiN}_{x}$ top layer. For both models an initial stress of $400 \mathrm{MPa}$ (either tensile or compressive) was assumed. (c) Line plots at the center of the model. The resulting strain in the Si cap is more tensile for a tensile strained $\mathrm{SiN}_{x}$ layers and less tensile for compressively strained $\mathrm{SiN}_{x}$. Here, a constant Ge composition of $40 \%$ inside the Ge islands was used for simplicity.

shows the in-plane strain for compressive and tensile stress in panels (a) and (b), respectively. Panel (c) shows the inplane strain along $z$-direction in the center of the islands, confirming the strange behavior. A more detailed investigation is currently under way, but it seems that the details of the shape of island and cap are important: the intuitive argument given above is true only in the case of flat layers. In our case, however, the cap follows the comparatively steep shape of the island, so that the nitride surrounding the "hill" in the $\mathrm{Si}$ cap actually pushes the Si inward and leads to a compressive strain. This inward push is larger than the outward push of the nitride directly above the Si hill, so that the nitride cap has the opposite strain effect than expected intuitively. As a side effect of these systematic simulations, we notice that using this approach high strain values up to $0.8 \%$ in the silicon capping layer can be achieved without introducing dislocations.

However, the XRD maps show that compressively strained nitride leads to larger relaxation of the SiGe islands, which is again in the direction one would have expected intuitively, so that there is a discrepancy here. Several effects could be responsible: The initial strain of the nitride layer depends sensitively on the growth conditions; the results could differ for flat substrates (samples used for curvature measurement to determine nitride initial stress) and substrates with islands and corrugations on top, leading also to inhomogeneous nitride thickness. In the determination of the shape of buried islands we rely on AFM images of uncapped ones grown under the same conditions. Although shape changes due to capping are small at the applied growth temperatures, they might still be important. Further studies are required to clarify the exact shape of the buried islands.

We would like to mention that ordered and capped SiGe islands offer a route for an increase in the hole mobility in MOS structures as well. In between two closely separated islands the $\mathrm{Si}$ channel is compressively stressed which leads to a splitting of the heavy and light hole bands and an increased hole mobility. ${ }^{23}$

In conclusion, we have investigated the Ge distribution in buried SiGe islands and the strain distribution in the islands and a Si cap layer. It turns out that vertical as well as lateral Ge gradients are present in our samples. We studied the influence of $\mathrm{SiN}_{x}$ layers on the strain distribution, important in a later stage on the route to devices in order to fix the strain in the $\mathrm{Si}$ even when the straining $\mathrm{SiGe}$ islands will be removed. Here, discrepancies between simulations and experiment still have to be clarified. However, we stress that as our investigations show, the tensile strain values in the $\mathrm{Si}$ cap can reach values up to $0.8 \%$ without introduction of defects and thus prove that the DOTFET concept is a viable route to further enhancement of Si-based devices.

\section{ACKNOWLEDGMENTS}

This work was supported by the FWF, Vienna (SFB025), and the EC d-DOTFET project (012150-2). The authors thank the staff at BW2 at Hasylab and at ID10B at the ESRF for their assistance during measurements and the group of $\mathrm{L}$. Miglio at the University of Milano Bicocca for their support on the 3D FEM simulations. Furthermore, they would like to thank M. Meduna for performing stress measurements on test series with $\mathrm{Si}$ wafers capped with $\mathrm{SiN}_{x}$.

${ }^{1}$ M. Ieong, B. Doris, J. Kedzierski, K. Rim, and M. Yang, Science 306, 2057 (2004).

${ }^{2}$ O. G. Schmidt and K. Eberl, IEEE Trans. Electron Devices 48, 1175 (2001).

${ }^{3}$ K. W. Ang, C. H. Tung, N. Balasubramanian, G. S. Samudra, and Y. C. Yeo, IEEE Electron Device Lett. 28, 609 (2007); R. A. Donaton et al., Tech. Dig. - Int. Electron Devices Meet. 2006, 465.

${ }^{4}$ www.phantomsnet.net/Picoinside/EUFET/Files/DDOTFET.pdf? Fundacion $=\mathrm{b} 55 \mathrm{~d} 4 \mathrm{ed} 01 \mathrm{c} 72 \mathrm{dc} 7 \mathrm{e} 010 \mathrm{ca} 51 \mathrm{f} 0 \mathrm{a} 5 \mathrm{ba} 80 \mathrm{c}$.

${ }^{5}$ E. S. Kim, N. Usami, and Y. Shiraki, Appl. Phys. Lett. 72, 1617 (1998).

${ }^{6}$ G. Jin, J. L. Liu, and K. L. Wang, Appl. Phys. Lett. 76, 3591 (2000). 
${ }^{7}$ O. G. Schmidt, N. Y. Jin-Phillipp, C. Lange, U. Denker, K. Eberl, R. Schreiner, H. Gräbeldinger, and H. Schweizer, Appl. Phys. Lett. 77, 4139 (2000).

${ }^{8}$ B. Yang, F. Liu, and M. G. Lagally, Phys. Rev. Lett. 92, 025502 (2004). ${ }^{9}$ J. J. Zhang, M. Stoffel, A. Rastelli, O. G. Schmidt, V. Jovanovic, L. K. Nanver, and G. Bauer, Appl. Phys. Lett. 91, 173115 (2007).

${ }^{10}$ Z. Zhong, A. Halilovic, M. Mühlberger, F. Schäffler, and G. Bauer, Appl. Phys. Lett. 82, 4779 (2003).

${ }^{11}$ Z. Zhong and G. Bauer, Appl. Phys. Lett. 84, 1922 (2004).

${ }^{12}$ M. Borgström, V. Zela, and W. Seifert, Nanotechnology 14, 264 (2003).

${ }^{13}$ A. Pascale, I. Berbezier, A. Ronda, and P. C. Kelires, Phys. Rev. B 77, 075311 (2008).

${ }^{14}$ C. Dais, H. H. Solak, Y. Ekinci, E. Müller, H. Sigg, and D. Grützmacher, Surf. Sci. 601, 2787 (2007).

${ }^{15}$ K. Kash, B. P. Van der Gaag, D. D. Mahoney, A. S. Gozdz, L. T. Florenz, J. P. Harbison, and M. D. Sturge, Phys. Rev. Lett. 67, 1326 (1991).
${ }^{16}$ K. Kash, J. M. Worlock, D. D. Mahoney, A. S. Godzd, B. P. Van der Gaag, J. P. Harbison, P. S. D. Lin, and L. T. Florenz, Surf. Sci. 228, 415 (1990).

${ }^{17}$ G. Vastola, A. Marzegalli, F. Montalenti, and L. Miglio, Mater. Sci. Eng., B (to be published), http://dx.doi.org/101016/j.mseb.2008.05.011.

${ }^{18}$ For a review, see J. Stangl, V. Holy, and G. Bauer, Rev. Mod. Phys. 76, 725 (2004), and references therein.

${ }^{19}$ C.-C. Wu and R. Hull, J. Appl. Phys. 100, 083510 (2006).

${ }^{20}$ G. Katsaros et al.,Surf. Sci. 600, 2608 (2006).

${ }^{21}$ M. S. Leite, G. Medieros-Ribeiro, T. I. Kamins, and R. S. Williams, Phys. Rev. Lett. 98, 165901 (2007).

${ }^{22}$ All FEM work presented here has been performed using the COMSOL Multiphysics Program Package, www.comsol.com.

${ }^{23}$ G. S. Kar, S. Kiravittaya, U. Denker, B.-Y. Nguyen, and O. G. Schmidt, Appl. Phys. Lett. 88, 253108 (2006). 